\title{
Real and reciprocal space order parameters for porous arrays from image analysis
}

\author{
Forrest H. Kaatz ${ }^{1 *}$, Adhemar Bultheel ${ }^{2}$, and Takeshi Egami ${ }^{3}$ \\ ${ }^{1}$ Department of Mathematics and Life/Natural Sciences, Owens Community College, Toledo, Ohio 43699 \\ ${ }^{2}$ Department of Computer Science, K.U.Leuven, Celestijnenlaan 200A, 3001 Heverlee, Belgium \\ ${ }^{3}$ Department of Materials Science and Engineering, and Department of Physics and Astronomy, The \\ University of Tennessee, Knoxville, Tennessee 37996; and Materials Science and Technology Division, \\ Oak Ridge National Laboratory, Oak Ridge, Tennessee 37831 \\ *electronic mail: fhkaatz@yahoo.com
}

Abstract

A real space technique based on the pair distribution function (PDF), and a reciprocal space method utilizing a two-dimensional fast Fourier transform (FFT) quantifies the order in porous arrays. Porous arrays fabricated from nanoscience technology are analyzed. The PDFs are fit with a series of Gaussian curves and the widths of the Gaussian peaks are used to model the linear strain in the array. An order parameter is defined from the PDF, and takes values from $[0,1]$, where the value one represents an ideal array. The radial distribution function (RDF) is also determined for the porous arrays. The FFT of the porous arrays is used to generate an order parameter as a ratio of intensity to the full width half maximum $(\sigma)$ of the peaks. Defined as relative intensity $I_{r} / \sigma$, this parameter takes values from $[0, \infty]$, where larger values represent more order in the array. We use a variety of available software to generate this data.

\section{Introduction}

The terms 'ordered' and 'highly ordered' frequently appear in the literature when authors describe the arrays they manufacture for nanoscale science and technology. This paper presents a real space and a reciprocal space technique to give quantitative measure to these terms. The Fourier transform of the real space coordinates enables us to calculate an order parameter as a ratio of intensity to the full width half maximum $(\sigma)$ of the FFTs. Defined as intensity $I_{r} / \sigma$, this parameter takes values from $[0, \infty]$ where larger values represent more order in the array.

Porous arrays can be fabricated on the nano-scale, thus permitting the growth and placement of nanowires, nanodots, nanotubes, and photonic crystals ${ }^{1}$ in two and three dimensions. They can be made with a high aspect ratio [1,2], allowing the formation of tubes and wires, or they may be thinned for the placement of dots. Typically, the structure is hexagonal, due to close packing of cells in two dimensions, but other types $[3,4]$ of arrays are possible.

Also applicable to this type of analysis is the $2 \mathrm{D}$ projection of $3 \mathrm{D}$ images. This takes into account electron and X-ray tomographic analyses, as well as TEM images of porous or semi-ordered materials, see Ref [5]. Another interesting use would be for images of surfaces cut slightly off axis (see the images from the three-dimensional holography work of Ref [6], which show an off-axis slice of a fcc holographic array).

A common method of array fabrication is that from anodized aluminum oxide $[1,2,7]$. In this method, a foil or thin film of aluminum is anodized in an acid to form 
porous aluminum oxide. To create an ordered array, frequently a two-step [8] procedure is used. The acid and voltage used determine the pore size and interpore distance [7]. The smallest pores are made using sulfuric acid while phosphoric acid can be used to create larger pores. The porous array is then filled with the material of choice to create nanowires or nanotubes, or the oxide may be thinned to form a template for the placement of nanodots. The technique of imprint lithography [1,2] has also been used to form dimples on an aluminum surface as a guide to pore generation. This creates a well ordered array, as is shown below.

Another method of creating nanoporous arrays is to use colloidal nanospheres [9] as a template for $2 \mathrm{D}$ or $3 \mathrm{D}$ pore formation. In this method, a dense array of latex spheres is packed in a thin 2D layer with a precursor solution that is subsequently calcined to form a porous array. The calcined array is then filled with the growth material of interest. In an alternate procedure, termed nanosphere lithography [4], the spheres are left in place and material is deposited in interstitial sites to form nanoarrays.

Order in arrays can result in physical properties not attainable from random or disordered arrays. Some examples include field emission from carbon nanotubes [10], magnetic arrays where the magnetism is affected by a demagnetization factor from neighboring magnetic nanowires [11], and in electronics, optoelectronics, sensing, highdensity storage, and ultra-thin display devices [12]. Carbon nanotubes need to be spaced for field emission properties, so that the effects of screening are minimized. Arrays of nanotubes were fabricated through the use of anodized aluminum oxide [13] and nanosphere lithographic techniques [14].

An order parameter has previously [15] been used to describe the order in fast Fourier transforms (FFTs) taken from nanoporous arrays. Denoted as (I/ $\sigma)$, it describes the ratio of the spot intensity I, and its full width at half-maximum (FWHM) $\sigma$ of the FFT images taken from scanning electron micrographs. This parameter $(I / \sigma)$ has been said [15] to be consistent with human recognition of the spatial order of holes. The order parameter $(\mathrm{I} / \sigma)$ takes values from $[0, \infty]$, and has been plotted vs. several variables of pore formation. Typically, one looks for a maximum in the plot to determine the optimum conditions for ordered pore formation. We use FFTs generated from images that previously had no FFT data to determine the order in the arrays. We note that this type of analysis can be used in conjunction with any imaging device not having FFT capability.

\section{Methods}

The technique of the atomic pair distribution analysis [16] can be applied to the study of porous arrays. The PDFs are created using Image SXM [17], which uses a bestfit ellipse to characterize particles (pores) in the image, and finds the corresponding coordinates of the centers. Using this information, Excel macros were written to calculate the PDFs. Suppose there are $N_{T}$ total cells (about 250 in a typical image), and $N$ centers from which the RDF and PDF are calculated $(N=19$ in our examples) $[18,19]$, and let $r_{i j}$ be the distance between the center of cell $i$ and cell $j$. To make the analysis scale independent, we use the average distance $\sigma$ of two neighboring cells as a unit and we shall in the rest of this paper assume that this rescaling has been done. This $\sigma$ is called the lattice constant of the array. Then the RDF is defined by 


$$
R(r)=\frac{1}{N} \sum_{i=1}^{N_{T}} \sum_{j=1}^{N_{T}} \delta\left(r-r_{i j}\right)
$$

where the $\delta(r)$ is a delta function. $N$ is less than $N_{T}$ to minimize edge effects in the calculated PDF and RDF, for a center near the edge would have only three or so neighbors. The $N$ centers should be chosen near the center of the image. There will be a peak around 1, the average distance between two neighboring cells, another peak around the average distance between the center of a cell and the centers of the next-to-closest ones. The PDF is then obtained by:

$$
\rho(r)=\frac{1}{2 \pi r} R(r)
$$

where $\rho(r)$ is the PDF.

To model the PDF and RDF we use a generalized form of a Gaussian function, i.e. a sum of eight Gaussians:

$$
\rho(r)=\sum_{i=1}^{8} a_{i} \exp \left[\frac{-\left(r-b_{i}\right)^{2}}{c_{i}^{2}}\right]
$$

where $a_{i}, b_{i}$, and $c_{i}$ are constants determined by the fit. Eight Gaussians is determined by the use of the Curve Fitting Toolbox (available as part of MATLAB), which is limited to eight Gaussians at a time. It is possible to fit more than eight only if a record is kept of previous fits and some are intentionally zeroed out in a subsequent fit.

The coordination number of a general peak is derived from the RDF as:

$$
N_{C}=\sum_{i=r_{1}}^{r_{2}} R\left(r_{i}\right)
$$

where $R(r)$ is the RDF, $N_{C}$ is the coordination number of a nearest neighbor site and $r_{1}$ and $r_{2}$ define the limits of the peak in the RDF. For the arrays we examine, $N_{C}=4$ for the first peak of the square array, and $N_{C}=6$ for the first peak of the hexagonal array. We find that the RDF drops to zero away from the center, in agreement with analysis of samples with finite sizes [20,21].

Now $\mathrm{R}(r)$ as we have defined it (and hence also $\rho(r)$ ) is only non-zero in a finite number of $r$-values, which can also conveniently be represented by a bin diagram. Suppose the bin size is 0.02 and that we consider 205 bins, which is enough to include the first 8 or 9 peaks, and includes some extra to get the right side of the last peak. We then define a disorder parameter as:

$$
\Delta \Phi=(0.02) \sum_{\xi=0.02}^{4.1} \rho\left(r_{\xi}\right)-(0.06) \sum_{\xi=n\left(r_{\xi}\right)} \rho\left(r_{\xi}\right) .
$$

Note that this disorder parameter is simply the sum of the product of the width of the bins (0.02) multiplied by the height of the PDF (the amount in the bin) and subtracting the amount in an ideal location. For very narrow bins these sums are integrals. Therefore, if all the contribution comes from the PDF in the ideal state $\Delta \Phi=0$. In general, $\xi$ is stepped by the bin width, 0.02. In the sum of the subtracted term, $\xi=n\left(r_{\xi}\right)$, the ideal eight or nine scaled nearest neighbors positions [18], and includes a bin above and below the (rounded) nearest neighbor location. The subtracted term is the amount in an ideal location and it is distributed over three bins. Three bins are used since simulations show 
that it is difficult to generate a PDF of an ordered array with $\Delta \Phi=0$ in a computer model unless one takes a bin above and below the nearest neighbor locations, $n(r \xi)$, some of which are irrational. This results in a rather sensitive order parameter

$$
\Phi=1-\frac{\Delta \Phi}{(0.02) \sum_{\xi=0.02}^{4.1} \rho\left(r_{\xi}\right)}
$$

where $\Phi$ takes values from $[0,1]$ and equals one when there is no disorder.

Reciprocal space data is often shown in experimental results [22-26] as a fast Fourier transform (FFT) image of the real space data. The intensity, $\boldsymbol{I}$, is determined from a Fourier transform of the data:

$$
\begin{aligned}
& I\left(\boldsymbol{k}_{x}, \boldsymbol{k}_{y}\right)=|\boldsymbol{F}|^{2} \\
& \boldsymbol{F}=\boldsymbol{X}\left(\boldsymbol{k}_{x}, \boldsymbol{k}_{y}\right)=\sum_{n_{1}=0}^{N_{1}-1} \sum_{n_{2}=0}^{N_{2}-1} x\left(n_{1}, n_{2}\right) W_{N_{1}}^{k_{k} n_{1}} W_{N_{2}}^{k_{y} n_{2}}
\end{aligned}
$$

where

$$
\begin{aligned}
& \boldsymbol{k}_{x}=0,1, \ldots, N_{1}-1 \quad k_{y}=0,1, \ldots, N_{2}-1 \\
& W_{N_{1}}^{k_{x} n_{1}}=\exp \left(-2 \pi i k_{x} n_{1} / N_{1}\right) \quad W_{N_{2}}^{k_{y} n_{2}}=\exp \left(-2 \pi i k_{y} n_{2} / N_{2}\right)
\end{aligned}
$$

where $\boldsymbol{x}\left(\boldsymbol{n}_{1}, \boldsymbol{n}_{2}\right)$ are the coordinates of the real space data, and $F$ is the 2D discrete Fourier transform. The 2D FFT is determined using MATLAB, which uses the FFTW3 routine [27]. The FFT as determined by MATLAB, is sensitive to pore position and number of pore sites. If the FFT of the image is $\mathrm{Y}=\mathrm{fft} 2(\mathrm{X})$, then this returns the two-dimensional discrete Fourier transform (DFT) of X, computed with a fast Fourier transform (FFT) algorithm, without zero padding. The resulting $\mathrm{Y}$ is the same size as $\mathrm{X}$.

The order parameter generated from this data is defined as:

$$
I_{r} / \sigma=\frac{I / I_{0}}{\sigma}
$$

where $I_{r}$ is the relative intensity with respect to the zeroth order peak, and $\sigma$ is the FWHM of the first order peak next to it (also equal to $c_{i}$ in equation (3) above). This order parameter takes values from $[0, \infty]$, and gets large for arrays with more order. Practically, however, since for an ideal array $I_{r}=I / I_{0}=1$, the largest the order parameter would be is near 100 or so, with $\sigma=0.01$, the FWHM equal to one pixel for an image of $100 \times 100$ pixels. We also note that the zeroth order peak is affected by finite size effects, so it seems unlikely one could have $I_{r}=1$ unless very large arrays are used.

\section{Results}

We model three arrays taken from previously published results. All of these were fabricated from anodized aluminum oxide (AAO). One, from Choi, et al. [1], was a hexagonal array made from a pre-patterned aluminum stamp and anodized in phosphoric acid. This produced an array used as a photonic crystal, with highly ordered pores. Another array, from Krishnan, et al. [3], was a square array made from AAO via interference lithography. This array is also fairly well ordered. The last example is a disordered array from $\mathrm{Li}$, et al. [5], also made from AAO, which we use to show the 
lower limit of the order parameters. We comment that the order parameter $I / \sigma$ has previously been used with FFT peak analysis as was done in reference [26].

The Choi and Krishnan PDFs are fit to a series of eight or nine Gaussian curves, and the fit is shown superimposed on the data in Figure 1.

Figure 1.A. An image of the array from Choi et al. (with permission, Ref. [1]) is shown with its PDF. A Gaussian fit is applied to the his the histogram of the PDF.
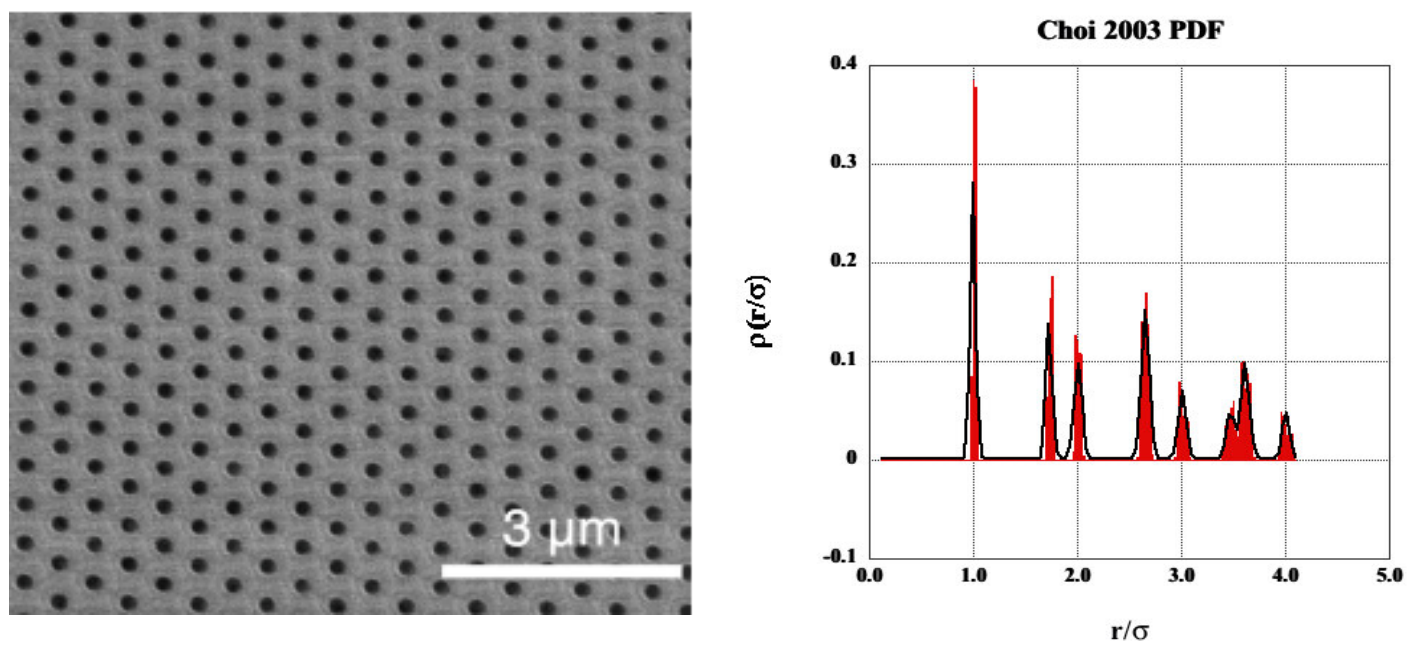

Figure 1.B. An image of the array from Krishnan et al. (with permission, Ref. [3]) is shown with its PDF. The PDF is fit with a series of eight Gaussian curves.
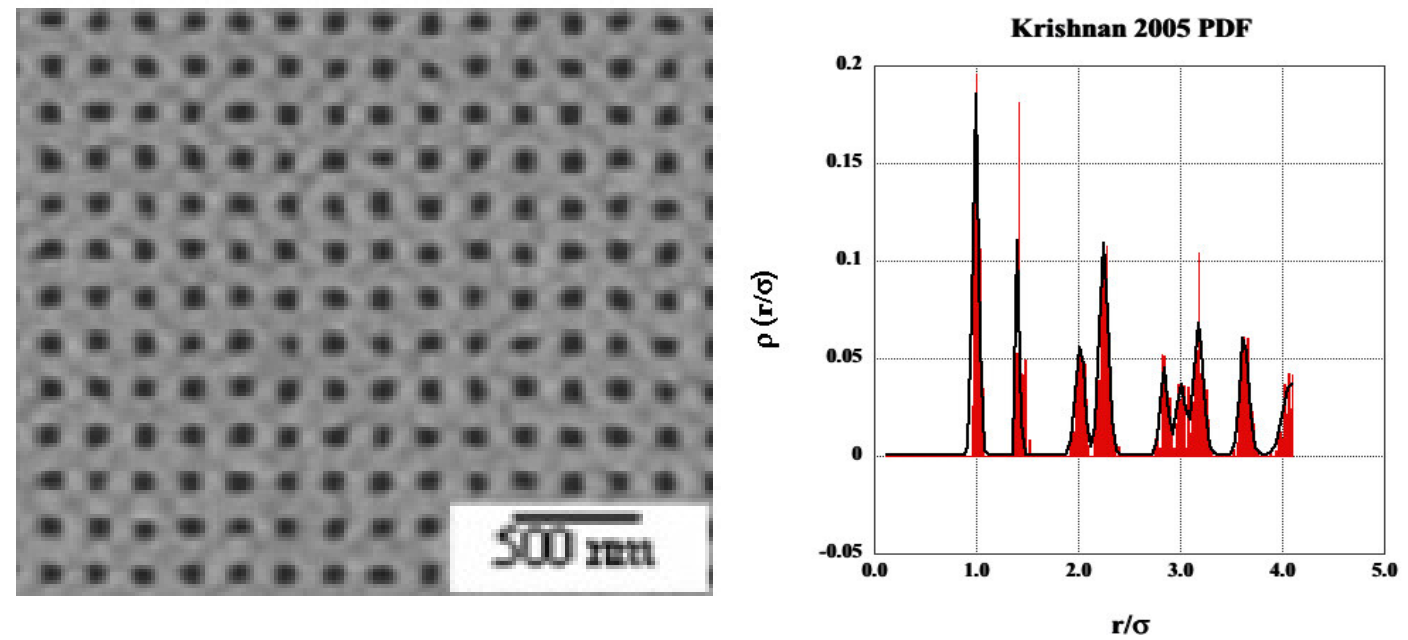
In the figure, we plot the normalized data, where $\sigma$ is the 'lattice constant' for the porous arrays. Since they are normalized, the peaks appear at the hexagonal nearest neighbors, i.e., $1, \sqrt{ } 3,2, \sqrt{ } 7,3$, etc., and the square nearest neighbors at $1, \sqrt{2}, 2, \sqrt{5}, \sqrt{8}, 3$, etc. The widths of the peaks are plotted against the normalized peak position in Figure 2. This shows the linear strain in the array as the distance from the center position increases. The error bars represent $+/-5 \%$ of the data values within $+/-1 \%$ of the standard deviation.

Figure 2 The Gaussian peak width showing the linear strain in the arrays is plotted versus normalized peak position.

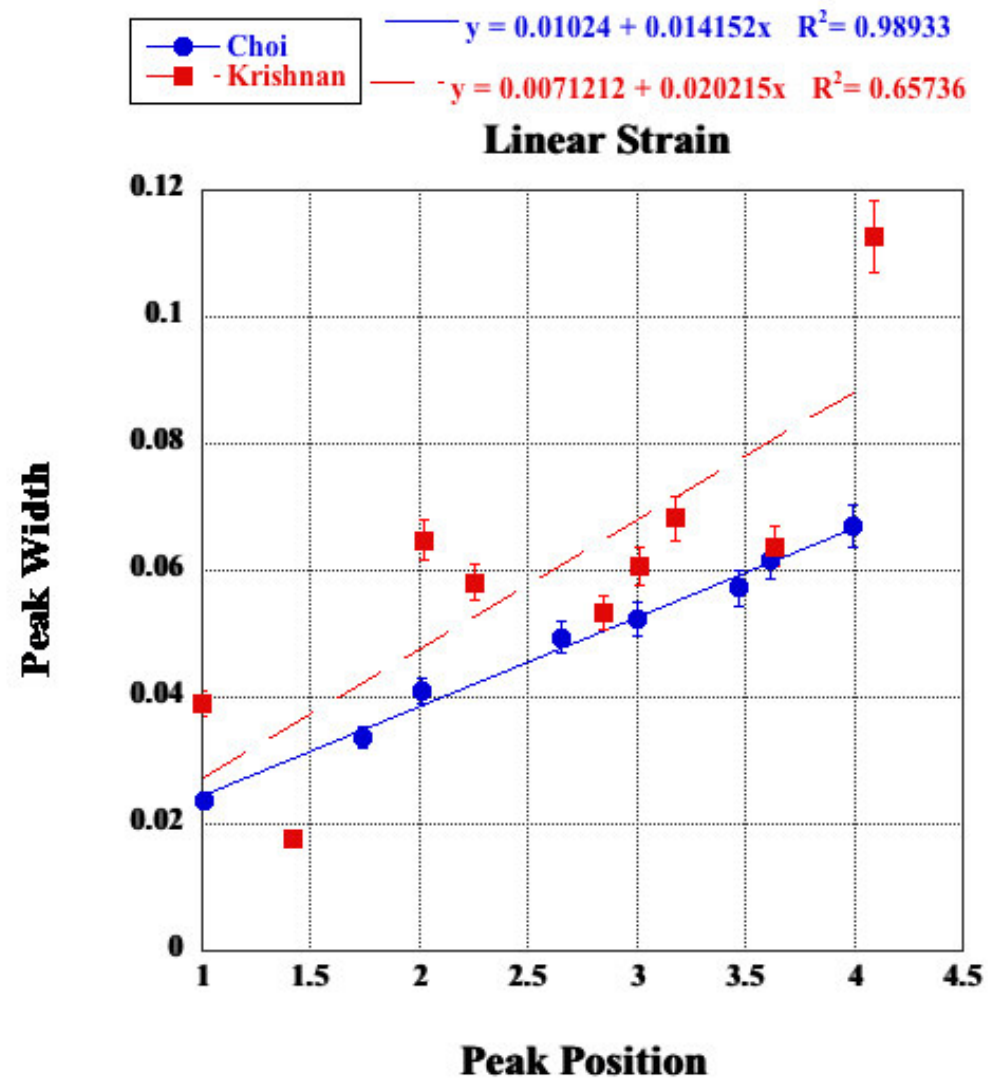

Table 1 lists the data for the real space order parameter $\Phi$ for the ordered arrays. The value of the order parameter is 0.69 for the Choi array and 0.55 for the Krishnan array. From the description of the order parameter, we see that the finite size of the image is built into the definition. In other words, it is possible to have a value $\Phi=1$ for a finite array, as long as the pore centers are in the proper locations. In this sense, the order parameter can be said to be robust. 
Table 1. the histogram of the PDF.

\begin{tabular}{|c|c|c|c|}
\hline Array & $\Phi$ & $\mathrm{RDF} \mathrm{R}^{2}$ & $I_{r} / \sigma$ \\
\hline Choi & 0.69 & 0.94 & 1.7 \\
\hline Krishnan & 0.55 & 0.89 & 1.9 \\
\hline $\mathrm{Li}$ & 0.14 & 0.37 & 0.03 \\
\hline
\end{tabular}

The RDF data for the three arrays is shown in Figure 3. From previous work on the crystallization of liquids in 2D [28], it has been determined that the packing density $\phi=$ 0.719 near the crystalline transition, and that the RDF approaches a value of one for large $\mathrm{r} / \sigma$. Figure 3 shows the RDF of the disordered array and that it approaches 0.5 for $\mathrm{r} / \sigma=4$. We also have that the packing density is $\phi=0.397$ for this array, from Image SXM. From the RDF plot and the packing density, we see that the disordered array is more liquid-like than the ordered ones, as is expected. Note that the packing density of the ordered arrays is not a valid quantity, since the pore size is controlled somewhat independently of the density, by the type of acid used for AAO formation.

Figure 3 RDF plots of the three arrays.

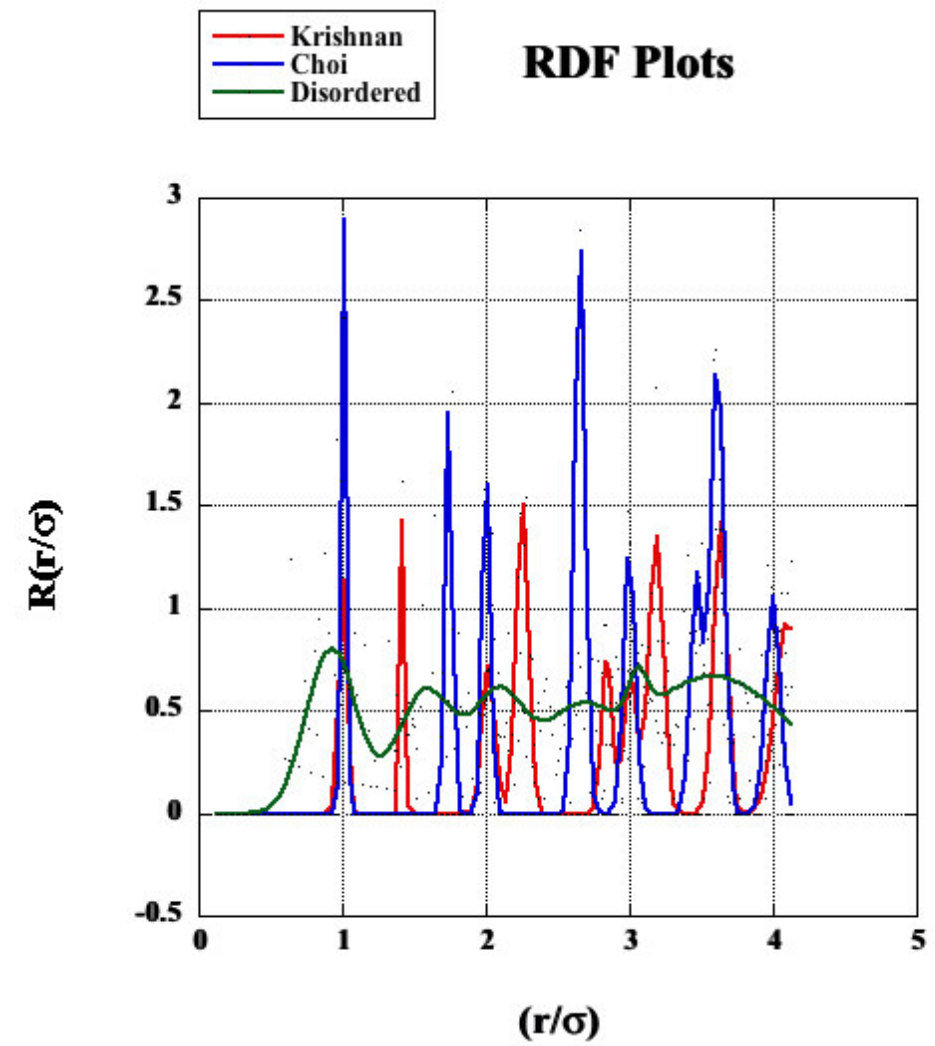

Figure 4 shows the arrays from Choi et al., Krishnan, et al., and a disordered array from $\mathrm{Li}$, et al. The top view is shown in the middle image and shows the FFT with sixfold or four-fold symmetry. Note that the FFT has the same symmetry as the 
Figure 4.A. Left panel: Real space figure from Choi, et al. Center panel: Diffraction pattern. Right panel: 3D plot of intensity vs. $\mathbf{k}_{\mathbf{x}}$ and $\mathbf{k}_{\mathbf{y}}$.
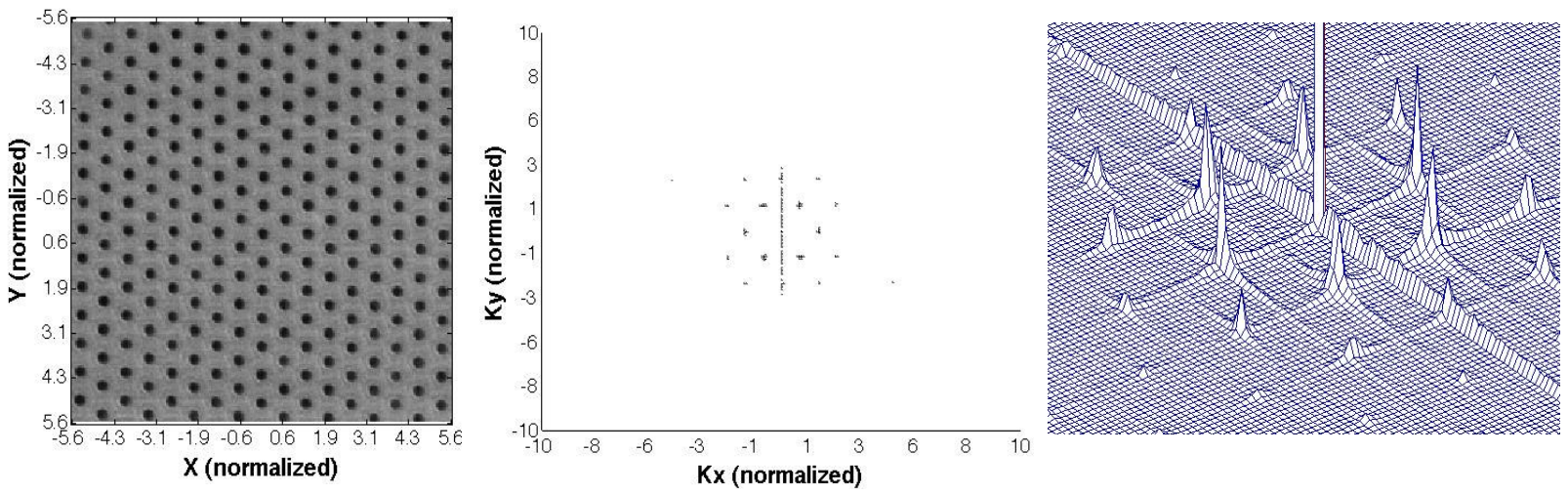

Figure 4.B. Left panel: Real space figure from Krishnan, et al. Center panel: Diffraction pattern. Right panel: 3D plot of intensity vs. $\mathbf{k}_{\mathbf{x}}$ and $\mathbf{k}_{\mathbf{y}}$.
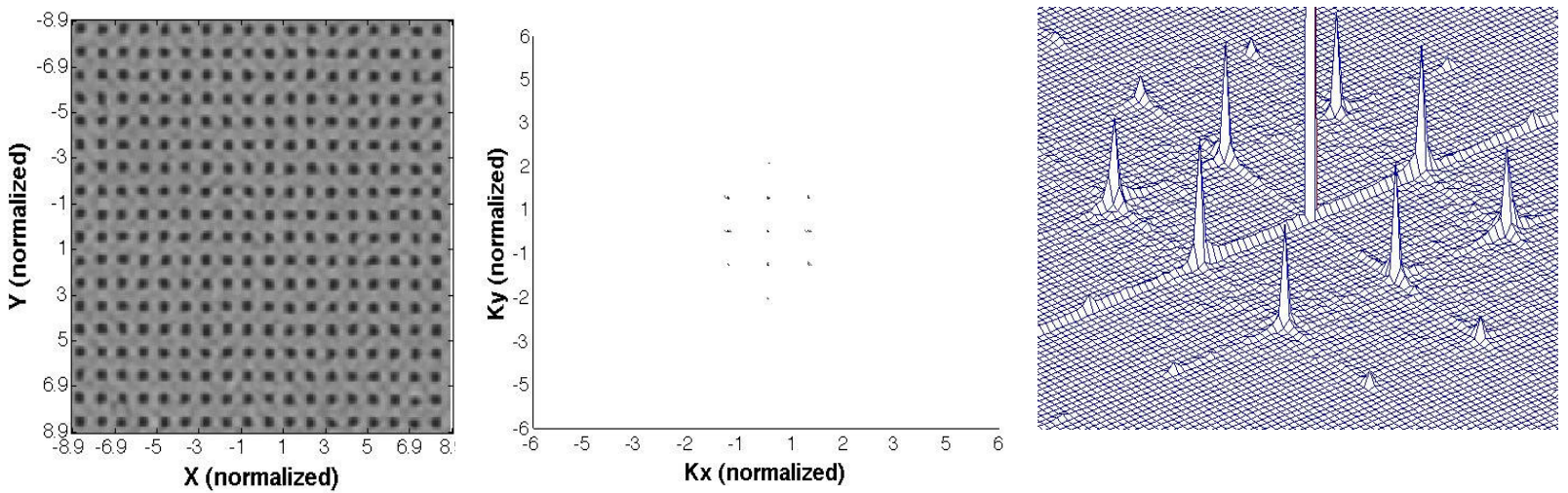

Figure 4.C. Left panel: Real space figure from Li, et al. (with permission, Ref. [7]). Center panel: Diffraction pattern. Right panel: 3D plot of intensity vs. $\mathbf{k}_{\mathbf{x}}$ and $\mathbf{k}_{\mathbf{y}}$.
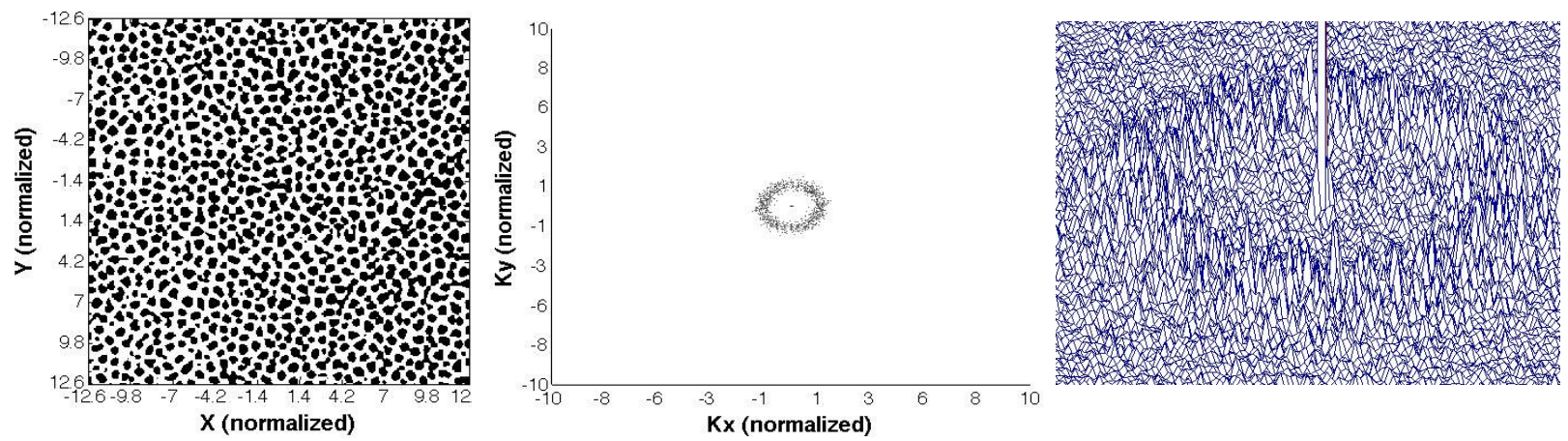
corresponding real space image. A 3D expanded view of the intensity of the FFT is shown versus $\mathbf{k}_{\mathbf{x}}$ and $\mathbf{k}_{\mathbf{y}}$ in the third panel. These plots are normalized so that the real space distance between pores is one unit. If the real space dimension is normalized then the reciprocal space unit will also appear at $\mathrm{K}=1$.

We show the intensity versus $\mathrm{K}$ space for the three arrays in Figure 5 . These are fit with a series of Gaussian curves with good $\mathrm{R}^{2}$ statistics. From the definition of the order parameter as $I_{r} / \sigma$, we calculate that it equals $0.03,1.7$, and 1.9 for the disordered array, the Choi array, and the Krishnan array, respectively. The first order peaks on either side of the main zeroth order one are used to calculate the order parameter. In the case where the peaks are not quite equal, we average over the two peak intensities. In the order parameter, $I, I_{0}$, and $\sigma$ are determined from the Gaussian fit obtained from the Curve Fitting Toolbox. We note that the amount of contrast in the original images may limit the quality of the corresponding FFT.

Figure 5.A. Intensity vs. K-space and the Gaussian fit from Choi, et al.

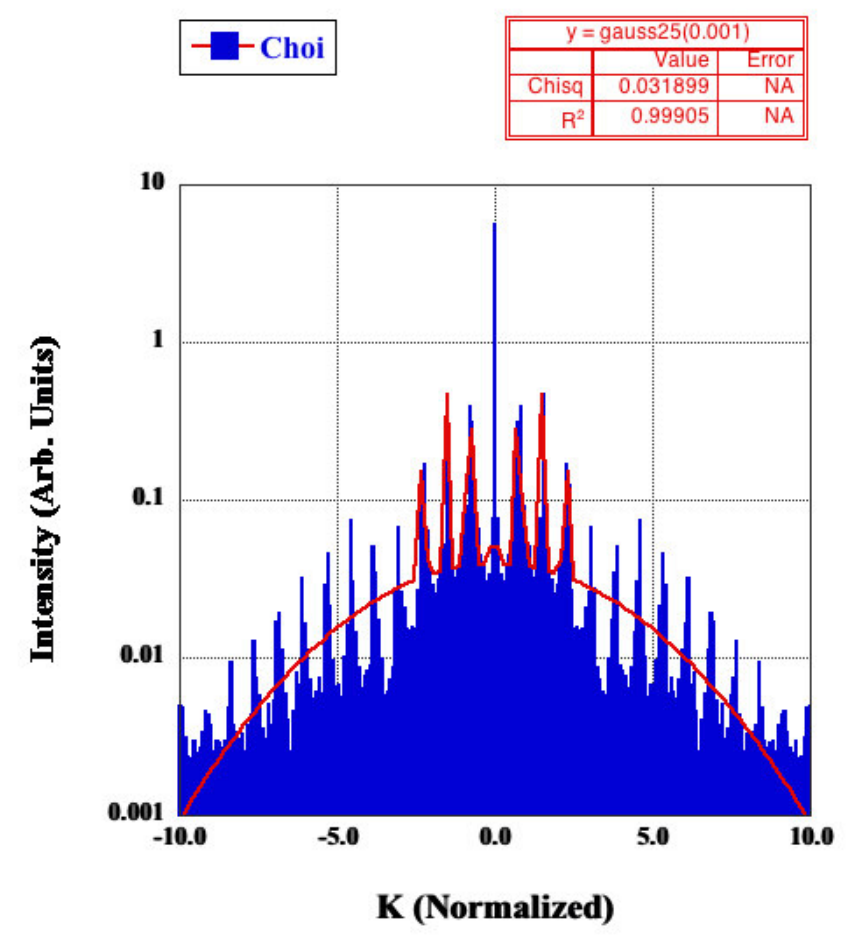


Figure 5.B. Intensity vs. K-space and the Gaussian fit from Krishnan, et al.

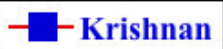

\begin{tabular}{||r|r|r||}
\hline \multicolumn{3}{|c|}{$\mathrm{y}=$ gauss31(0.01) } \\
\hline & Value & Error \\
\hline Chisq & 0.0039778 & $\mathrm{NA}$ \\
\hline $\mathrm{R}^{2}$ & 0.99987 & $\mathrm{NA}$ \\
\hline
\end{tabular}

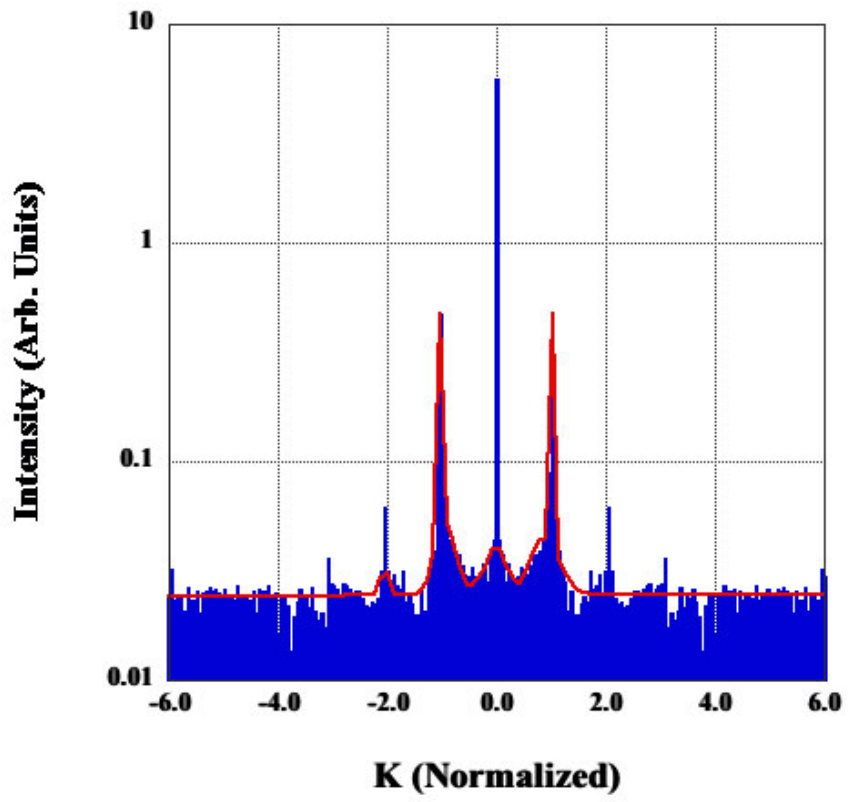

Figure 5.C. Intensity vs. K-space and the Gaussian fit from Li, et al.

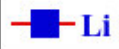

\begin{tabular}{||r|r|r||}
\hline \hline \multicolumn{3}{|c|}{$\mathrm{y}=$ gauss22(0.01) } \\
\hline & Value & Error \\
\hline Chisq & 0.013957 & NA \\
\hline $\mathrm{R}^{2}$ & 0.99896 & $\mathrm{NA}$ \\
\hline
\end{tabular}

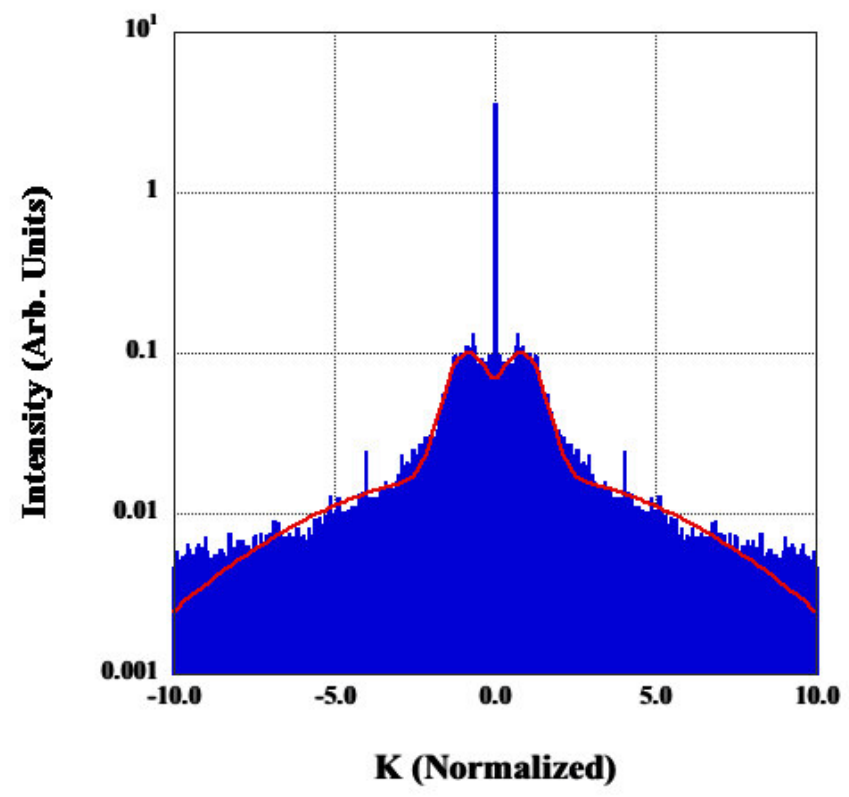




\section{Discussion}

A real space and a reciprocal space order parameter has been calculated for these arrays. In our opinion, $\Phi$ may be a more accurate parameter since the size of the arrays is built into the definition. In principle the definition could be modified to accommodate a larger $\mathrm{r} / \sigma$. A calculation of $\Phi$ for ideal arrays has been determined in Ref. [18] and equals one as expected. The reciprocal space order parameter, $I_{r} / \sigma$, is affected by the intensity of the zeroth order peak and is not corrected by a finite size effect.

In the analysis for the coordination number, $N_{C}$, the fact that the calculated sums over the first peaks in the RDF are the same as the observed coordination number (4 or 6 ) for the square or hexagonal arrays provides evidence that the RDF and PDF have been correctly determined. It is true that there is some arbitrariness in the definition of $\Phi$, in that the spacing between bins is 0.02 and that $r / \sigma$ is determined out to 4.1. The choice of the bin spacing is determined primarily by the spacing in the arrays and provides an adequate density of bins. In principle this could be changed in future work to accommodate different images.

In our analysis, we use Image SXM and the Curve Fitting Toolbox associated with MATLAB. The PDF and RDF have been fit with a series of Gaussian curves, using the Curve Fitting Toolbox. The correctness of this model is shown in Ref. [19], where a bin diagram and Gaussian curves are shown to be good approximations to the delta functions in the definitions of the PDF and RDF. In the cases of Figs. 1, 3, \& 5, the data has been fit in Kaleidagraph, since a macro fitting more than eight Gaussians may be necessary. Although not included in this analysis, the uniformity of the pores can be derived from Image SXM, since it will also give the average areas of the pores and the elongation of the ellipse for the enclosed pore area.

\section{Conclusion}

In summary, a quantitative analysis has been undertaken for porous arrays. Both real space and reciprocal space analysis has been done. The strain in the arrays is modeled from the width of the PDF peaks using a linear fit. The measured differences in the order parameters originate from the alternate ways they are defined. The order parameter $\Phi$ is a real space measure, essentially related to the height and width of the PDF histograms, while $I_{r} / \sigma$ is related to its reciprocal space definition. Since the length scale is normalized, the methods described can be applied to any image and a corresponding order parameter can be calculated. The use of procedures applicable to general image analysis has determined order parameters for real space images and these methods are useful for determining the order in any porous array. The methods described in this paper make use of widely available software to analyze porous arrays.

\section{Acknowledgments}

An iMac@2.4 GHz running Mac OS X 10.5.5 was used. Image SXM is a free download for the Mac OS available from Ref. [17]. MATLAB, the Curve Fitting Toolbox, Excel, and Kaleidagraph complete the software tools necessary to create and analyze the data. 


\section{References:}

1. Choi, J., Luo, Y., Wehrspohn, R.B., Hillebrand, R.B., Schilling, J. and Gösele, U. (2003) J. Appl. Phy. 94(8), 4757-4762.

2. Asoh, H., Nishio, K., Nakao, M., Tamamura, T., and Masuda, H. (2001) J. Electrochem. Soc. 148(4), B152-B156.

3. Krishnan, R., Nguyen, H.Q., Thompson, C.V., Choi, W.K., and Foo, Y.L. (2005) Nanotechnology 16, (2005) 841-845.

4. Hulteen, J.C., Treichel, D.A., Smith, M.T. Duval, M.L., Jensen, T.R., and Van Duyne, R.P. (1999) J. Phys. Chem. B 103, 3854-3863.

5. Blanford, C.F., Carter, C.B., and Stein, A., Journal of Microscopy (2004), 216(3), 263287

6. Campbell, M., Sharp, D.N., Harrison, M.T., Denning, R.G., Turberfield, A.J., (2000) Nature, 404 (6773) 53-56.

7. Li, A.P., Müller, F., Birner, A., Nielsch, K., and Gösele, U. (1998) J Appl. Phys. 84, 6023-6026.

8. Masuda, H. and Fukuda, K. (1995) Science 238, 1466-1468.

9. Sun, F., Cai, W., Li, Y., Cao, B., Lei, Y., and Zhang, L., (2004) Adv. Funct. Mater. 14(3), 283-288.

10. Shang, X.F., Wang, M., Qu, S.X., Zhao, R., Zhou, J.J., Xu, X.B., Tan, M.Q., and Li, Z.H. (2008) Nanotechnology 19, 065708.

11. Sellmyer, D.J., Zheng, M., and Skomski, R. (2001) J Phys Condens Matter 13, R433.

12. Lei, Y., Cai, W., and Wilde, G. (2007) Progress in Materials Science 52, 465-539.

13. Li, J., Papadopoulos, C., Xu, J.M., and Moskovits, M. (1999) Appl. Phys. Lett. 75, 367.

14. Park, K.H., Lee, S., Koh, K.H., Lacerda, R., Teo, K.B.K., and Milne, W.I. (2005) J. Appl. Phys. 95, 024311.

15. Shingubara, S., Okino, O., Sayama, Y., Sakaue, H., and Takahagi, T. (1997) Jpn. J. Appl. Phys. 36, 7791-7795.

16. Egami, T. and Billinge, (2003) Underneath the Bragg Peaks: Structural Analysis of Complex Materials, S.J.L. Amsterdam: Pergamon.

17. Barrett, S. November (2008). The website of Image SXM is at http://www.liv.ac.uk/ sdb/ImageSXM/

18. Kaatz, F.H. (2006) Naturwissenschaften 93, 374-378.

19. Kaatz, F.H., Bultheel, A., and Egami, T. (2008) Naturwissenschaften 95, 1033-1040.

20. Kodama, K., Iikubo, S., Taguchi, T., and Shamoto, S. (2006) Acta Cryst. A62, 444453.

21. Mason, G. (1968) Nature (London) 217, 733-735.

22. Kashi, M.A., and Ramazani, A. (2005) J. Phys. D: Appl. Phys. 38, 2396-2399.

23. Ba, L., and Li, W.S., (2000) J. Phys. D: Appl. Phys. 33, 2527-2531. 
24. Rao, Y.L., Anandan, V., and Zhang, G. (2005) J. of Nanoscience and Nanotechnology 5, 2070-2075.

25. Kashi, M.A., Ramazani, A., Rahmandoustand, M., and Noormohammadi, M. (2007)

J. Phys. D: Appl. Phys. 40, 4625-4630.

26. Sulka, G.D. and Parkoaa, K.G. (2006) Thin Solid Films 515, 338 - 345.

27. Frigo, M. and Johnson, S.G. (2005) Proc. IEEE, 93(2), 216-231.

28. Reis, P.M., Ingale, R.A., and Shattuck, M.D. (2006) Phys. Rev. Lett. 96, 258001. 